\title{
3D Printed Bioconstructs: Regenerative Modulation for Genetic Expression
}

\author{
Pravin Shende ${ }^{1}$ (D) $\cdot$ Riddhi Trivedi $^{1}$ \\ Accepted: 7 January 2021 / Published online: 16 January 2021 \\ (C) The Author(s), under exclusive licence to Springer Science+Business Media, LLC part of Springer Nature 2021
}

\begin{abstract}
Layer-by-layer deposition of cells, tissues and similar molecules provided by additive manufacturing techniques such as 3D bioprinting offers safe, biocompatible, effective and inert methods for the production of biological structures and biomimetic scaffolds. 3D bioprinting assisted through computer programmes and software develops mutli-modal nano- or micro-particulate systems such as biosensors, dosage forms or delivery systems and other biological scaffolds like pharmaceutical implants, prosthetics, etc. This review article focuses on the implementation of 3D bioprinting techniques in the gene expression, in gene editing or therapy and in delivery of genes. The applications of 3D printing are extensive and include gene therapy, modulation and expression in cancers, tissue engineering, osteogenesis, skin and vascular regeneration. Inclusion of nanotechnology with genomic bioprinting parameters such as gene conjugated or gene encapsulated 3D printed nanostructures may offer new avenues in the future for efficient and controlled treatment and help in overcoming the limitations faced in conventional methods. Moreover, expansion of the benefits from such techniques is advantageous in real-time delivery or in-situ production of nucleic acids into the host cells.
\end{abstract}

Keywords Nucleic acids $\cdot$ DNA $\cdot$ Bone $\cdot$ Extrusion $\cdot$ Scaffolds $\cdot$ Bioinks

\section{Introduction}

The aspect of modification or structural changes such as replacing, splicing, silencing, editing, controlling or inactivating of a defective gene or delivery of a new gene is known as gene therapy and it shows various applications in cancers, tumours, infectious diseases and genetic disorders. [1] Novel nucleic acid or gene delivery systems based on recombinant DNA technology are, therefore, researched upon to identify the target location and transfer the required gene into the cell. The vehicles of delivery or vectors of gene delivery are mainly of two types, 1) viral vectors (adenoviruses, lentiviruses, heplex simplex viruses, retroviruses, etc.) deliver nucleic acids into target cells that are unable to replicate by themselves, and offer high transduction and gene expression, whereas 2) non-viral vectors directly inject gene and gene conjugates into the cell

Pravin Shende

shendepravin94@gmail.com

1 Shobhaben Pratapbhai Patel School of Pharmacy and Technology Management, SVKM'S NMIMS, V. L. Mehta Road, Vile Parle (W), Mumbai, India via physical (Electro- or sono-poration, microinjections, gene gun, magnetic or hydrodynamic delivery, etc.) or chemical methods such as nanocariers (nanoparticles, liposomes, dendrimers, polymers or oligonucleotides, etc.). [2] Processes such as introduction of RNA interference (RNAi) used for gene silencing, and other nucleic acids such as shRNAs, siRNA or miRNA opens up potential avenues in the targeted treatment of variety of disorders such as viral infective diseases, neuroblastomas, eye disorders, etc. [3, 4] Gene therapy also focuses on the use of stem cells that are genetically modified either as a therapeutic agent or a gene delivery system in wound therapy, skin regeneration or against scar formation using signals to modify molecular and cellular activities and mechanisms of the wound or target tissue. [5, 6] However, gene therapy is a still a complex field of medicine which further requires extensive research as many limitations are faced in treatment and delivery of gene to the target site. Viral vectors develop issues like immunogenicity (produce immune responses) or toxicity in the body, similarly non-viral vectors may demonstrate low transfection issues, while both vectors used in delivery may show off-target effects, lack in efficiency, purity and cannot contain higher concentrations or sizes of the required gene (especially limited DNA-carrying ability). [2, 7, 8] The clinical success of gene 
therapy is still to be established fully as control release kinetics and efficacy in delivery of macromolecules (e.g. polypeptides) are a major challenge. Gene therapy, therefore, requires for a superior method of gene transfer that surpasses these challenges of the conventional delivery systems.

On the other hand, 3D printing technology provides realtime, flexible, stable, diverse and quick result with advancements in novel drug delivery systems and dosage forms by sequential layering. This technology helps in improving the conventional dosage forms, targeting the drug to the active site and controlling of pharmaco-kinetic and -dynamic parameters. It further offers new potentials in medicine by aiding in the preparation of personalized and controlled release therapeutic systems with fabrication of compliant procedures for patients. [9-13] 3D printing technology enables the production of scaffolds that can incorporate nucleic acids, growth factors, stem cells, etc. and offers a platform for desired (localized, safe and sustained) release of such molecules in the body. Furthermore, enhanced or modified gene expression achieved via 3D scaffolds also opens avenues in tissue engineering, wound healing and skin regeneration.

\section{D Printing (3DP) and Bioprinting (3DBP)}

$3 \mathrm{D}$ printing (3DP) is a form of additive manufacturing technique to construct a three dimensional structure wherein a $3 \mathrm{D}$ printer is used to deposit or join successive layers of input materials on a desired substrate. [14] Similarly, assembly of body parts such as tissues and organs or the fabrication of biological elements with the help of such 3D printing processes and layer by layer addition of bioinks is known as 3D bioprinting (3DBP). [15]

The method or mode of action of 3D printing and bioprinting are different, even though their concepts are similar since both the approaches employ the extrusion of compounds and substances to develop a scaffold or structure. The primary difference arises from the types of materials used for sequential deposition, where 3D printers use inorganic substances, while bioprinters use bioactive agents, biomaterials or biomimetic molecules. Traditional 3D printing technique refers to the use of materials such as polymers, metals, alloys, plastics, ceramics, resins, etc. [16] which are deposited on the substrate in a certain sequence (layer-by-layer) to create desired constructs such as implants, surgical instruments, pacemakers, bone plates, etc. In case of $3 \mathrm{D}$ bioprinting, which is an extension of $3 \mathrm{D}$ printing, is aimed at printing biomimetic tissues or cell models such as blood vessels, skin tissues, multicellular structures that can mimic the structure and function of target tissues. Bioinks used in bioprinting are organic molecules, mainly living cells, and other natural or synthetic biomaterials such as gelatin, alginate, polymers, hyaluronic acid, collagen, etc. [15] They also include liquid, paste or gel-based scaffolds that act as substrates or glue on which the cells grow (e.g. cell-laden hydrogels, cell suspensions) [17]. Bioinks are selected carefully for the bioprinting process so as to maintain the integrity of the printed system. The type of bioink used depends on the nature and characteristics of product or tissue. For example, alginate or hyaluronic acid bioinks enable the generation of nanocellulose hydrogels which may be used for bioprinting stem cells and other growth factors that lead to cartilage matrix production, while collagen is used in production of cell-laden structure applicable for regeneration of various tissues like adipose stem cells. Similarly, silk-type bioink used in biocompatible gelatin and glycerol hydrogels promoted cellular infiltration and tissue integration [15].

This technology renders many opportunities in the field of biology, medicine and in healthcare industry such as development of drug delivery systems and production of biomimetic implants, scaffolds, prosthetics or bioelectronics and biosensors. [18] 3D printing combined with the benefits of nanotechnology provides with novel techniques for preparation of personalized or customized therapeutic nanosystems, biomaterials, smart devices and for improvement in delivery of drugs. [16] The advantages of $3 \mathrm{D}$ printing include enhancement in functional structures of dosage forms or nanocarriers (nanoparticles, polymers, hydrogels, etc.), regeneration of essential cells, preferred drug release profile, pre-clinical testing and diagnostic avenues, enhanced functional properties such as geometry and anisotropy, less time consuming, economic and cost-effective manufacturing, high production capacity and yield and tailormade and patient compliant therapeutic systems. $[14,17]$ The production steps involved in $3 \mathrm{D}$ printing are extensively reduced as compared to the conventional methods of preparation of a dosage form which leads to an increase in quality of the delivery system or dosage form. $[14,16,18]$

The procedure of $3 \mathrm{D}$ printing is relatively simple and involves the collection of data from various health monitoring devices such as CT scanners, MRIs along with the computeraided designing software that helps in establishing prototypic models based on the morphology of the models. [17] In bioprinting, this step is crucial in developing an anatomical structure so as to resemble the original biological systems. Data in the model is individually transferred to the printing device and later the materials are processed, evaluated and automatically printed layer-by-layer in real-time according to the model plan which then solidifies into the actual structure.

The various 3D printing approaches are direct energy deposition, powder bed fusion, binder deposition, inkjet or penbased printing, vat polymerization, stereolithography, material jetting, extrusion, etc. However, bioprinting techniques may be based on main approaches of: 1) extrusion which involves pneumatic methods for continuous formation of paste filaments from hydrogels or melting polymers, such methods can use high density cells and highly viscous bioinks, however risk the distortion of cell structure and loss in viability, 2) inkjet or 
droplet-based method which involves ejection of droplets, rather than filaments through a thermally applied nozzle, 3 ) stereolithography (photopolymerization) and laser-induced stacking of printed materials onto a substrate. [19-21] Factors such as temperature, pressure, speed required to be controlled and optimized, especially in bioprinting based on extrusion methods. The main printing techniques for 3D printing and 3D bioprinting, their characteristics and important materials for each approach are summarized in Table 1 and the overview of the printing processes are depicted in Fig. 1.

\section{Applications of 3DP for Genetic Modulation}

$3 \mathrm{D}$ printing and bioprinting have extensive uses in the field of complex formation through colloidal self-assemblies [22], tissue or bone regeneration [23-27], neuroblastoma cell culture systems [28], fabrication of nerve conduit or implant engineering [29-31], alignment of muscle cells [32], in vaccine delivery [33], molecular diagnostics [34], etc. 3DBP technology holds a great potential for gene delivery into the defective cells for various diseases, in tissue engineering and regeneration medicine and especially in treating bone defects, wherein the specific nucleic acid is precisely placed into the $3 \mathrm{D}$ printed tissue, organ or a scaffold (bone or muscle implants) for treatment. This is also true for stem cells, enzymes, growth factors and other bioactive elements that increase the gene expression since they are assembled into the 3D structures layer by layer. Some of these 3D printing applications are summarized in Fig. 2. Similarly, evolution of stem cell or other cell therapy, nanotechnology and molecular medicine have accelerated in the last two decades with novel gene delivery systems

Table 1 3DP vs 3DBP - Summary of the main printing techniques involved in both approaches

3D printing (3DP) $\quad$ 3D bioprinting (3DBP) References

Inkjet-based technique

Binder jetting/Drop-on-powder: Selectively dispenses liquid binder solution onto a power bed.

Similar to 3DP in method, however, no powder bed is used and

Directing an acoustic wave/ electric heating of print head to generate pressure pulses to force droplets from nozzle.

Materials used: Polymer, ceramic, glass

Improved printing, controllable volume of liquid droplets, particle size (50 to $100 \mu \mathrm{m})$

Disadvantage: may involve toxic organic binders, and requires thermal post-treatments - may denature biomolecules, lack in precision.

\section{Fused deposition modeling} binding liquids are replaced with crosslinked cell-laden hydrogels.

High precision, high speed, ability to form heterogeneous structures with multiple types of cells.

Disadvantage: Limited resolution, low cell density, narrower range of materials as compared to 3DP.

Thermoresponsive/ Thermoplastic polymers extruded through heated nozzle by rollers and deposited layer by layer, and fused with previous structures.

Materials used: polycaprolactone (PCL), PLGA, etc. and their composites.

Does not use potentially-toxic solvents.

Involves multiple prinitng heads and can deliver several materials simultaneously.

Disadvantage: requires supporting structures, slow cooling and hardening of thermoplastic polymers - may significantly slow the printing process.

Selective laser sintering (SLS)

\section{D extrusion bioprinting}

Pneumatic/Pisoton/Screw driven extrusion dispensing system to obtain continuous filaments in a three-dimensional pattern. Includes a temperature-controlled material-handling component and a receiving platform.

Suitable for materials with high viscosity and high cell density.

Materials: Thermoplastic polymers with high viscosity - low biocompatibility, low cell viability.

Natural Polymers (collagen, gelatin, fibrin, alginate and silk) - poor printability and low resolution.

\section{D laser-assisted bioprinting (LAB)}

Laser beam on powder layer to sinter particles into a designed pattern and repeated layer by layer.

Materials: Hydroxyapatite (HAp), tricalcium phosphate, PCL and polyvinyl alcohol (PVA)

High mechanical strength and low porosity

Disadvantage: requires high temperatures - not applicable for delivery of cells or growth factors.

Pulsed laser beam used on cell-laden hydrogels fixed onto a donor slide (coated with laser absorbing materials) inducing cell-laden droplets which are propelled onto a receiving substrate (coated with biomaterials/medium for cell adhesion).

Microscale resolution and fast deposition.

No shear stress observed (due to nozzle pressure) - preserves cell shape.

Disadvantage: photo-induced cell damage, crosslinking required to maintain shape, high cost.

\section{Stereolithography (SLA)}

Light-mediated (UV/IR/laser beams) chemical reaction to form 3D scaffolds from photocurable liquid polymer/resin.

Materials: Photosensitive polymers/resins

Well-defined geometry and sub-micrometer resolution.

Disadvantage: slow, resins used are non-biomimetic, includes cytotoxic photo-initiators. 
Fig. 1 Schematic presentation for 3D bioprinting methods

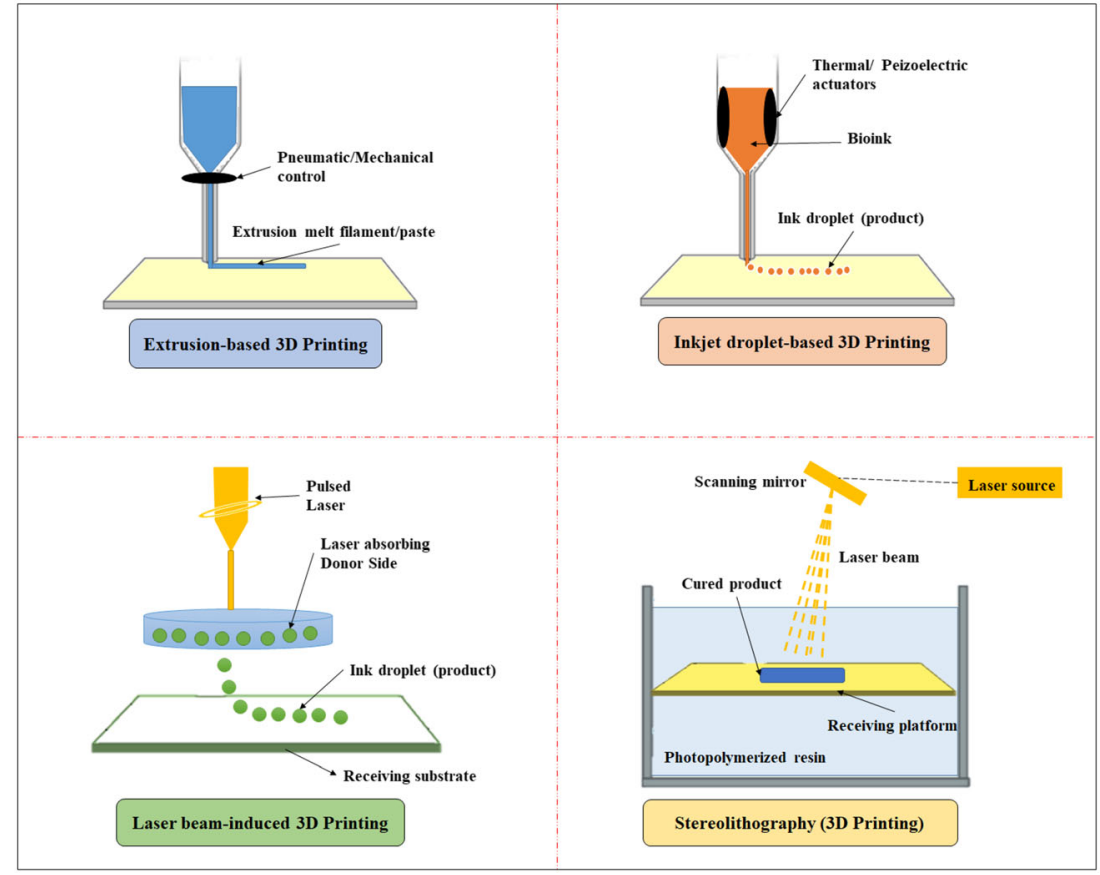

developed for efficiently transfecting the gene into the host cell. Nanocarriers, vectors and stimuli-responsive cargo or conjugation systems are developed with advanced release properties that help in easy transfer of gene in various editing techniques. Nanocarriers used in combination with 3D printed scaffolds are used for their individual characteristics, delivery of genes and related molecules and controlled release properties. [35] The methods of preparation for gene modulating and gene therapeutic scaffolds are summarized in Fig. 3. An example of gene delivery this is the study that fabricated a $3 \mathrm{D}$ printed scaffolds for miRNA delivery. Here, poly-lactide (PLA) 3D printed scaffolds were developed via Tinkercad software application and extrusion-based method. These scaffolds were then coated with Rhodamine-labelled fluorescent PAMAM dendrimers. Finally FAM-mir-503 (miRNA gene) was incubated with these functionalized PLA scaffolds. The transfection of HeLa cells on the PLA scaffolds were evaluated and cell proliferation was measured. The confocal microscopy images showed that the internalization of fluorescent dendrimer derivatives facilitated cell proliferation on its surface and acted as novel non-toxic delivery vectors for transferring miRNAs into human cells in in-vitro studies [36].

Applications of 3DBP were also seen in fabrication of actual cells, tissues and organs as a tissue engineering approach and for development of biomaterial scaffolds with cell seeding precision. For example, Chinese Hamster Ovary (CHO) cells were 3D bioprinted with thermal inkjet technology where printhead consisted of narrow nozzle channel (diameter: $48 \mathrm{~mm}$ ) to eject cells and further, these cells were analysed for cell viability, presence of membrane damages (caused due

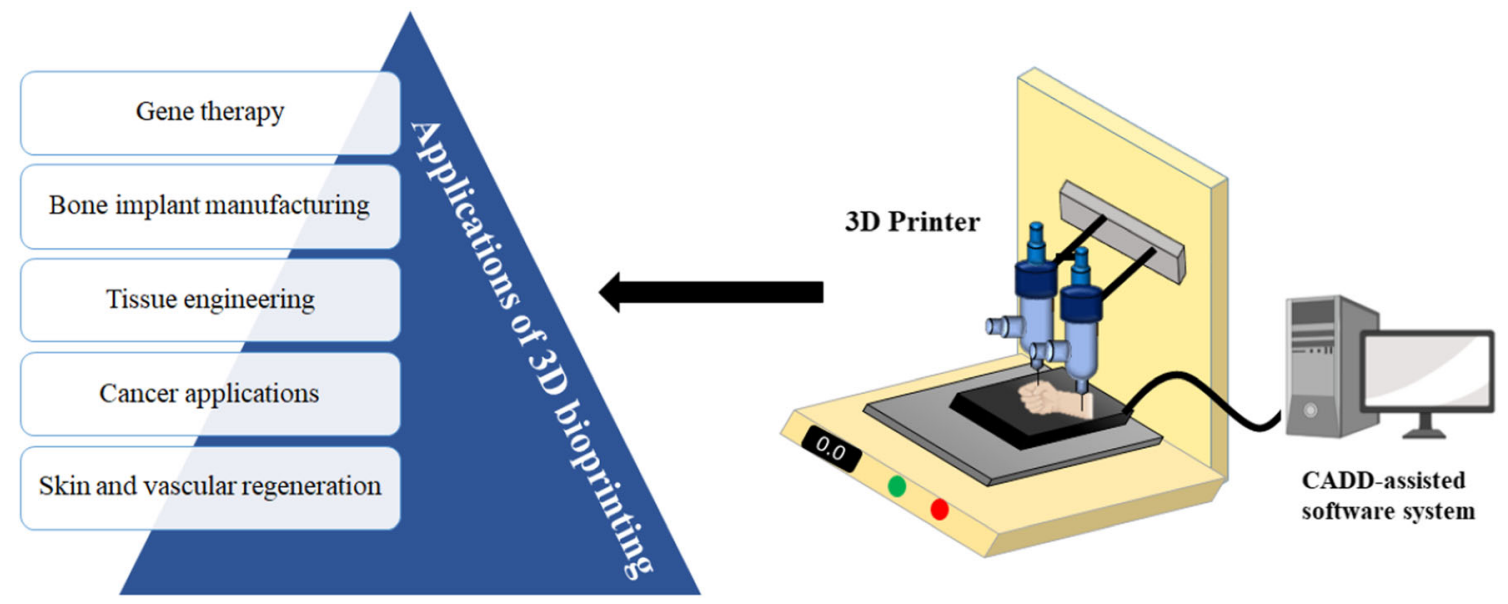

Fig. 2 Applications of 3D bioprinting 

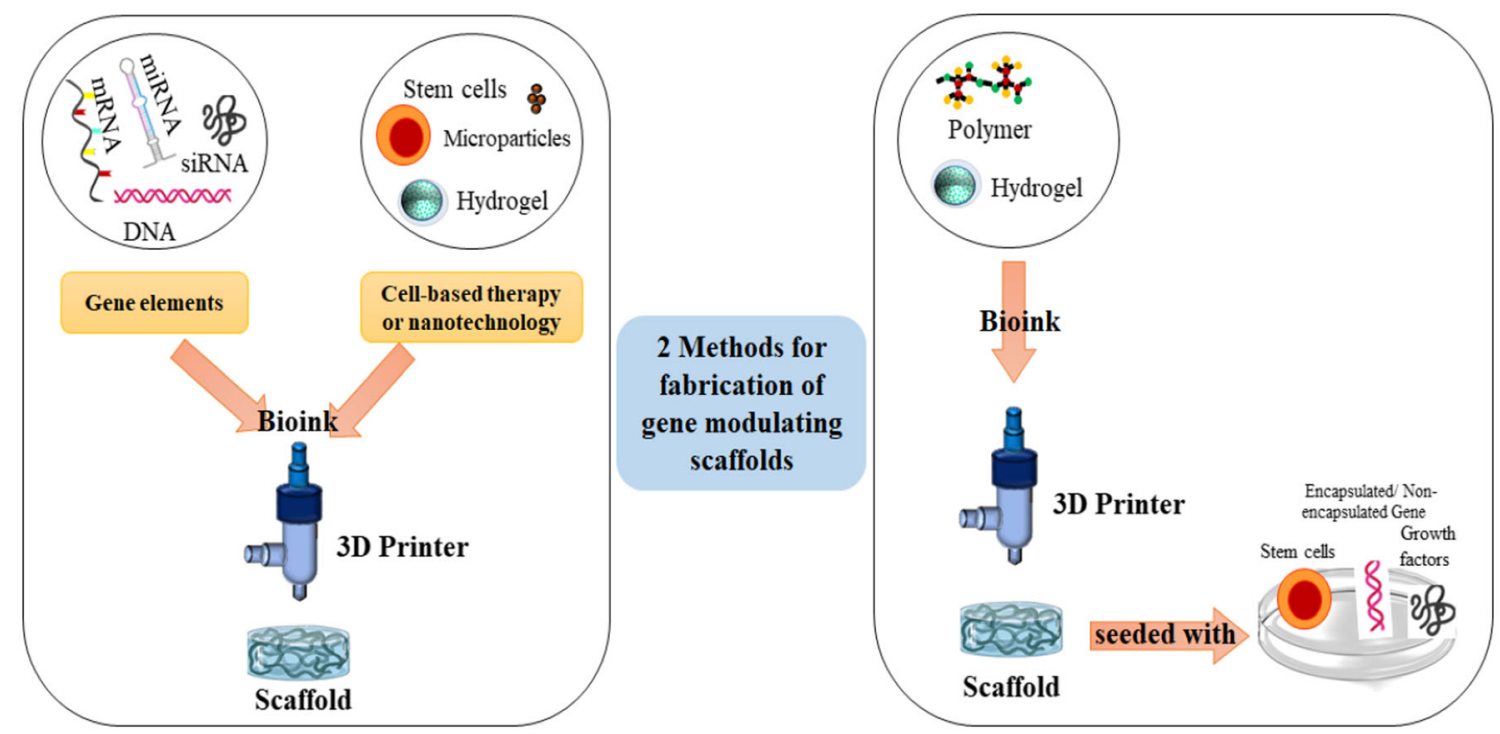

Fig. 3 Methods of preparation for gene modulating scaffolds

to thermal heat or stress from the inkjet-based system), and apoptosis. The results demonstrated cell viability of $\sim 89 \%$ and apoptotic cells $\sim 3.5 \%$, while the transient pores in the cell membrane developed during printing process were repaired after $2 \mathrm{~h}$ of printing. A notable feature of this study is the delivery of targeted genes like green-fluorescent protein (GPA) DNA plasmids to CHO-S cells by co-printing method. The transfection efficiency was found to be above $\sim 30 \%$ for cells printed with GFP-plasmids, whereas GFP expression was not found in unprinted cells mixed with plasmids. It was also noted that the transient pores in cell membranes helped in the effective delivery of gene microparticles, which further induced growth of engineered tissues. [37] A similar study used Porcine Aortic Endothelial (PAE) cells to evaluate gene transfection and cell-based delivery of inkjet-based 3D printed scaffolds. Here, the in-vitro transfection efficiency was obtained over $\sim 10 \%$ while the post-transfection cell viability was over $\sim 90 \%$. Furthermore, alternate printing of fibrinogen and thrombin, followed by direct co-printing of PAE cells and plasmid DNA in-vivo into subcutaneous tissues of athymic mice resulted into a 3D cuboid-based fibrin gel constructs with transfected gene. Targeted delivery of genetically modified PAE cells into 3D fibrin gel scaffolds was demonstrated by GFP expression and thus showed a potential in developing 3D scaffolds in-situ for cell-based gene therapy. [38]

Another study used mRNA therapy for repairing calvarial bone defects by $3 \mathrm{D}$ printing of hybrid scaffolds as a delivery system. This study transfected photoactivated miR-148bconjugated silver nanoparticles on bone marrow stem cells and then loaded the cells into collagen crosslinked 3D scaffolds comprised of poly (lactide-co-glycolide) (PLGA), polycaprolactone (PCL) hydroxyapatite (HAp). A personalized 3D printer (Multi-Arm BioPrinter) was used to mechanically extrude the scaffold frames. The transfected miRNA cells yielded: 1) higher expression of osteogenetic markers such as RUNX2 and biomineralization bone sialoprotein (BSP), 2) enhanced bone formation, and 3) higher bone mineral density $(\sim 34 \%)$ as compared to the non-transfected cells. This structure was fabricated to overcome the challenges such as disorganised tissue interfaces, biological instability, short half-life, poor tissue integration, etc. faced in conventional craniofacial gene therapy. [39] Similarly, applications of personalized and local delivery programming factors for bone repair can be seen in another experiment wherein polymeric (PLGA/PEG) microparticles $(\leq 50 \mu \mathrm{m})$ were loaded with transcription factor GET-RUNX2. These microparticles were mixed with temperature sensitive materials (Pluronic F-127, etc.) and extrusion-printed via RegenHU 3D Discovery 3D printer, so that these scaffolds can transform into bone-like lattice structures when exposed to body temperature. Later the effects of mesenchymal stem cells were co-printed with 3D scaffolds containing microparticles. The in-vitro studies demonstrated that the seeded stem cells induced higher osteogenesis action on exposure to the transcription factor due to the sustained release profile of RUNX2 from the encapsulated microparticles. [40] On the other hand, 3D printed scaffolds are also capable in modulating gene expression as proved by an experiment, wherein hydrogels were developed for regeneration of bone tissue with the help of biocompatible bioinks, namely gelatin and alginate. The conformational changes in the fabricated scaffolds such as size, porosity and mechanical properties altered the osteogenic gene expression of MC3T3E1 cells. [41]

Non-viral gene vectors incorporated in bioprinted structures as a strategy for regenerative medicine and tissue engineering were prepared in a study. Here, plasmids (pDNA) were incorporated in nano-hydroxyapatite solution to form complexes, and then a gene activated bioink was prepared 
by mixing these complexes with RGD- $\gamma$-irradiated alginate solution. Then, transfection of these complexes to pigderived mesenchymal stem cells (MSCs) was achieved. Finally, this gene activated bioink was 3D printed with into a construct by 3D Discovery multi-head system and fused deposition technique, which co-printed the bioink and melted poly-caprolactone into mechanically stable scaffolds. The invitro studies also demonstrated better osteogenesis effect of MSCs due to the effective co-delivery of pDNA encoding therapeutic genes - Bone Morphogenetic Protein 2 (BMP-2) and Transforming Growth Factor (TGF- $\beta 3$ ). The gene activated scaffold maintained the gene expression (for over 14 days) and increased vascularity (by 12 weeks) with uniform bone deposition. The in-vivo study results over a period of 1 month also showed significantly increased levels of mineral deposition. Thus, this study revealed a tailor-made and 'point-of-care' method of gene transfection in osteogenesis and gene delivery. [42] Similarly, 3D printing also facilitated the development of other genetically modified bioinks that are either pore-forming hydrogels that show rapid transfection or solid bioinks that show sustained effects. These bioinks further led to the formation of mechanically enhanced constructs that offered effective delivery of peptide based pDNA genes and enabled the establishment of spatially complex tissue structures. [43] Application of 3D bioprinting was also observed in congenital hip joint dysplasia-induced articular cartilage injury characterized with the decreased expression levels of Growth Differentiation Factor (GDF5). In this study, constructs based on genetically inspired polymeric 3D bioprinted scaffolds were fabricated, wherein the growth factor gene was conjugated on rabbit-derived bone marrow stem cells which was then converted into a cell-laden hydrogel and printed along with PCL. Computer generated tissue models were used for controlling the organ printing united system (OPUS). The results exhibited $\sim 95 \%$ cell viability with an enhanced repairing effect in-vivo in rabbit knee cartilage and further showed even tissue regeneration. [44] Non-viral vector delivery of gene (DNA) encoding an osteogenic agent called bone morphogenetic protein-2 (BMP-2) is used in the regenerative medicine in order to form bone in-vivo. This study prepared a gene activated 3D hydrogel for inducing bone growth and blood vessel formation in the body in case of bone fractures or other defects. Here, BMP-2 transfected on goatderived multipotent stromal cells (MSCs) were combined with alginate and calcium phosphate particles to fabricate 3D structures via BioScaffolder system based on 3D fibre deposition technique. The scaffold provided sustained production and release of pBMP-2 for 5 weeks and also showed a greater in-vitro osteocalcin expression from porous bioprinted scaffolds $(\sim 70 \%)$ than from solid scaffolds $(\sim 50 \%)$ and as compared to conventional controls $(<2 \%)$. On the other hand, staining analysis showed negative results suggesting that there was no apparent bone formation in-vivo at the end of 6 weeks.
The study concluded that the negative results were caused by higher alginate concentration used in the scaffold that resulted in smaller pore size and slow and untimely release of BMP-2, thereby demonstrating that mechanical aspects of $3 \mathrm{D}$ printed scaffolds play a major role in gene delivery and expression. [45]

Cell-based gene delivery by fabrication of 3D scaffolds are explored for the treatment of acquired or hereditary diseases and established their applications in tissue re-engineering and treatment of cancers. A study developed micro/macroporous poly-L-lactide (PLLA) scaffolds which were seeded with mesenchymal stromal cells (MSCs) derived from murine bone marrow. These MSCs were genetically engineered to secrete erythropoietin (EPO). The scaffolds were fabricated with solid free-form technology using 3D plotting and pore leaching techniques, which provides interconnected pores on the scaffolds. The microporosity of the scaffolds promoted oxygen and nutrient transport thereby enhancing cell survival and viability, whereas the macropores increased cell migration. The high surface area, porosity and the staggered offset plotting pattern of the scaffolds increased cell attachment and proliferation and metabolic activity for over 2 weeks and enhanced the delivery of therapeutic proteins and soluble gene products. Genetically modified MSCs promoted the sustained release of soluble gene product (EPO protein) which also increases with porosity of the scaffolds. [46]

Primary or secondary metastatic tumors which metastasized to bone are resected during surgery, however, they leave a void and may lead to recurrence of the tumors due to the residual tumor. Scaffolds developed by additive manufacturing (3D printing) that fill such void and enable sustained or prolonged release of drugs may be able to destroy the remaining cancerous cells and hence such structures are explored in chemotherapy. A study developed a dual-delivery 3D printed PCL scaffold functionalized with chitosan/siRNA nanoparticles and doxorubicin, wherein siRNA was used to induce gene silencing for preventing drug resistance from the tumors and also in inducing a synergistic effect with doxorubicin for cancer cell death. Here, the scaffold showed a burst release which was then followed by slow release of siRNA which induced sequence specific gene silencing that was sustained for 7 days in non-small cell lung carcinoma (H1299) and glioma (U251) cells. Furthermore, the toxicity studies showed that the synergistic cytotoxicity of chitosan nanoparticles with low concentrations of doxorubicin. It was induced in a siRNA sequence independent manner in lung cancer cells as compared to non-coated groups. This synergism was speculated due to increase in the uptake of doxorubicin via cellular membrane destabilization and/or through induction of apoptosis or necrosis due to the combination of drugs. [47] 
Gene therapy for the treatment of vascular diseases entails the use of expression vectors encoding angiogenic factors. Angiogenic gene therapy involves the transfer of genes, encoding angiogenic factors and thus promotes the formation of new vessels (angiogenesis). The main genes used for the preparation of expression vectors are Vascular Endothelial Factor (VEGF) and Fibroblast Growth Factor (FGF). [48] Various studies utilized angiogenic factor VEGF to form a vascular network in the tissue as an application of tissue regeneration. A study encapsulated VEGF into nanoparticles by complexing with dextran sulphate and coacervation by using chitosan as a polymer. These nanoparticles were then incorporated into two types of 3D matrices - PLGA scaffolds and Matrigel ${ }^{\mathrm{TM}}$ hydrogels. The efficiency of VEGF was improved through encapsulation method and controlled release pattern from these 3D implants and also improved angiogenesis in-vivo. Hydrogel-loaded encapsulated VEGF increased angiogenesis $\sim 5-7$ fold as compared to hydrogel-loaded free VEGF, while $\sim 3.5$ fold increase in angiogenesis was found in encapsulated VEGF-loaded scaffolds as compared to un-encapsulated VEGF loaded into PLGA scaffolds [49]. Although this study worked without $3 \mathrm{D}$ printing technology, Matrigel ${ }^{\mathrm{TM}}$ (only for mice) [50, 51] and PLGA [52] scaffolds are used as bioinks for bioprinting applications and hence similar applications can further be explored in the field of bioprinting for fabrication of implants to release the growth factors and for tissue regeneration. Similar platforms for regenerative medicine applications and techniques for gene delivery from hydrogels are explored, in case of heparin-chitosan nanoparticles with PEG hydrogels for lentivirus delivery and for expressing VEGF to promote angiogenesis. The lentivirus-functionalized PEG hydrogels were prepared by dissolving PEG-acrylate with a photoinitiator, frozen and exposed to UV light (for crosslinking PEG solution). These hydrogels were then incorporated with heparin-chitosan nanoparticles for immobilizing lentivirus within the porous PEG hydrogel structures. The binding and retention of lentivirus in this formulation led to a sustained and substantial increase in transgene expression in in-vitro as well as in-vivo studies and also demonstrated improvement in vascular growth. [53]

Moreover, along with gene delivery, the use of 3D printed gene-based nanosystems can be used in establishing stimuliresponsive nucleic acid-functionalized biosensors or as systems to localize DNA signals. An example is the use of DNA-functionalized bioinks used in additive nanomedicine manufacturing. This approach was based on the costeffectiveness and simple production methods wherein the DNA provides dynamic and pattern-forming techniques. Here, DNA strands were incorporated within hydrogels with the help of a droplet extrusion-based 3D printer (Ultimaker Original+) assisted with a python script reading software to localize DNA sequences and program its diffusion properties. The blend of gelatin, alginate and agarose functionalized with ssDNA via click chemistry were loaded in the print head and extruded through the nozzle on glass slides in the preparation of hydrogels [54]. Another example, of gene-functionalized $3 \mathrm{D}$ printing, is the study in which DNA-DNA interaction was used as a 'smart-glue' for adhesion of microparticle assembly in a colloidal gel in 3D extrusion. The structural integrity of this gel and assembly of the microparticles in the host cells was maintained solely by DNA-DNA interactions. Here, first the suspension of DNA-conjugated polystyrene microparticles were prepared which were later loaded in a computercontrolled and modified Replicator 3D printer to form a selfassembled colloidal gel with different 3D shapes. The shape and pore sizes and rheology of this colloidal system was maintained via the complementary DNA linker sequences present as a glue between successive microparticles. DNA adhesives or connectors in 3DP provide their applications in designing of complex colloidal structures. [55] The production of siRNA and miRNA-loaded nanocarriers for treatment of diseases also considered as an important application of 3DP in gene delivery. One study developed 3D printed microfluidic chips as an indirect method of producing siRNA-polymer nanocomplexes for downregulation of proteins and silencing of genes. Different geometries, hydrodynamics and channel designs of the printed chips provide with difference in efficiencies of the nanocomplexes. Here, microfluidic chips were 3D printed via the stereolithography technique using Accura 25 (resin) as the bioink and COMSOL Multiphysics 5.3a module for designing. Further, nanocomplexes were fabricated by injecting siRNA solution through central inlet, the PAMAM polymer dendrimer through side inlets and their flow rates were controlled and both the solutions were mixed inside the microchips. Optimization of hydrodynamic flow with respect to different geometry of the microfluidic chips was carried out with in-silico simulations. The desirable characteristics of the formed siRNA nanocomplexes such as charge and size were dependent on the microfluidic chip geometry and could be modulated with change in channel width, channel size and angle spacing. Hence, production of nanocomplexes via microfluidics showed better reproducibility, enhanced time and quality control due to automatic procedures as compared to conventional methods. [56] Similar applications of 3D printed scaffolds, implants and reengineered tissues along with CAD-assisted devices are established to deliver either genes (siRNA, mRNA and artificial oligonucleotides) for gene editing and therapy or to transfer elements (proteins, enzymes, vaccines) in order to modulate the gene expression. Conversely, genetically modified biological elements may be used as bioinks in the stabilization of $3 \mathrm{D}$ printed scaffolds, e.g. elastin-like recombinamers were genetically modified and used as bioinks for bioprinting of loaded cells. This biomimetic bioink provided conducive environment for cell growth and proliferation. [57] The overall picture of studies demonstrating gene therapy or modulation through $3 \mathrm{D}$ printed scaffolds is depicted in Fig. 4. 

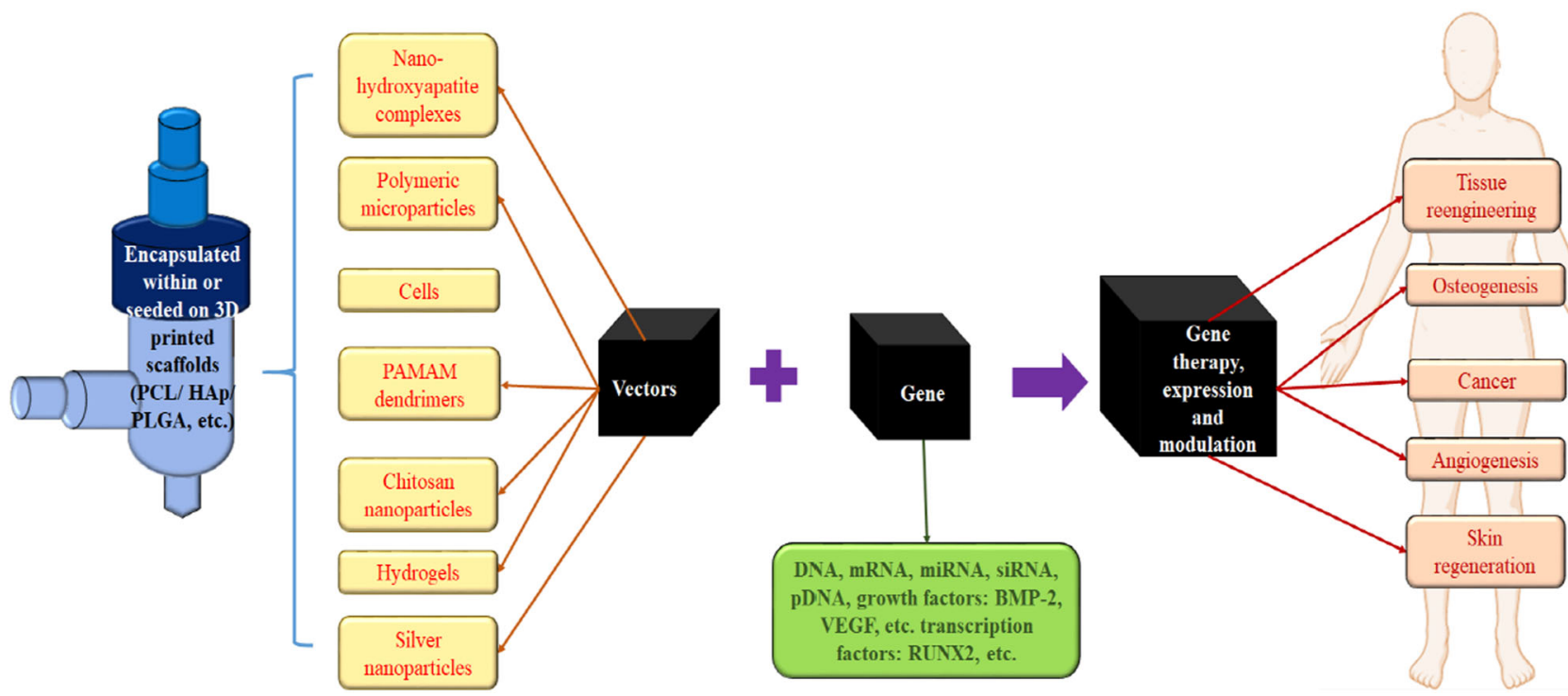

Fig. 4 Overall summary of 3D printed scaffolds used in gene therapy

\section{Future Trends}

\section{Direct Gene-Printing}

The relationship between a molecular structure and its function is extremely important in 3DBP and the lack of this knowledge leads to errors in manual handling of 3DBP devices and failure of the printed biological structures. The development of dynamic 3D printed models in depended on the information of their biochemistry which needs to be considered while building such machines. Similarly, the molecular information provided by nucleic acids, their interactions and specific mechanisms and functions may lead to better delivery systems. Current DNA synthesizing methods may face several limitations ranging from the length of the oligonucleotides that create errors in the process of correct genome sequencing, low yield, time consumption, failure in adhesion of DNA sequences, toxic chemical reagents used in synthesis and use of toxic solvents and environmental working conditions of the lab. Direct gene printing is one of the fields that is gaining increasing focus as it offers avenues for convenient production of nucleic acids avoiding the limitations faced in their manufacturing. Synthetic generation of DNA sequences may be possible for medical treatments and other healthcare applications. Cambrian Generics, a biotechnology company based in San Francisco, used a laser-based technique to print (or sort) DNA. The DNA is conjugated on metal beads and are scanned and evaluated by a computer to find the correct sequences for synthesis. The correct DNA sequence is then bombarded by a computer-assisted laser beam which is further collected on glass plate, while the impact of this beam causes the beads to carry the correct sequences. This technique can be extremely useful in producing billions of strands at once. It might be used to develop ideal or exceptional gene sequences to further enhance or modify the functions of the body, for example, engineering of microbial genome for development of new medicines or changing the protein sequence in the treatment of cancer. [58-60] The applications can further be extrapolated in the future for gene editing wherein the faulty gene can be sorted and removed or the corrected functional gene can be synthesized. Synthetic Genomics® built an automated 3D printer (BioXp ${ }^{\mathrm{TM}} 3200$ system) for benchtop synthesis and cloning of linear DNA fragments (into a plasmid vector) with an overall efficiency rate of approximately $\sim 83 \%$. This system generates DNA clones overnight from customized oligonucleotide pools prepared from the desired sequence information and later fed into the system for the cloning process. Here, the gene sequence is first submitted into the software after which the custom reagents are selected and the system is loaded and then run to sort and clone the DNA fragments. It automatically performs manual functions such as pipetting, mixing, thermal cycling, purification and storage. [61-63] Gene editing technologies such as the CRISPR/Cas9 system is a modification of bacterial immune system that uses a single guide ( $\mathrm{sg}$ ) RNA to further activate the Cas9 endonuclease to the site of action. [64] This further helps in cleavage of DNA at the targeted site. Further advancements in technologies like CRISPR/Cas9 system that is used in genome engineering and its incorporation into 3DP applications may emerge as a robust methods for gene therapy [65]. Similarly, mobile molecule mRNA printers or 'RNA microfactories' are said to be developed by Tesla Inc. in order to build mRNA-based vaccines for COVID-19. $[66,67]$

\section{In-Situ Printing}

$3 \mathrm{D}$ bioprinting techniques face limitations with respect to lack of knowledge on tissue regeneration and structural 
parameters, proper sterilization procedures, and skill to create new tissues or organs in-vitro. With further advances in 3D printing with the incorporation of computerized algorithms, bioinks and materials along with knowledge in mechanisms and functions of body organs, it is possible to establish in-situ 3D bioprinting or 'in-vivo bioreactor' applications. [68] It is a minimally invasive technique of constructing a functional biological system (tissue/organs) directly at the damaged or wounded anatomical site via a machine or device and have been especially useful in skin regeneration and bone or cartilage repair [68-70]. This technology has many benefits such as it may not require to reproduce the micro-environmental conditions, is simple to use, can be cost-effective and directly produce cells or tissues at the site of action. These techniques include 3D bioprinting via 1) robotic arm approach consists of real-time printing through a 3 -axis movable device which is a computer-aided real-time tissue designing technique wherein less human contact is required (although under surgeon's supervision), or 2) the handheld approach, which is used by the surgeon, involves preparation of tissues through a flexible and portable device consisting of a unit that directly deposits biomaterials on the living cell. These in-situ printing techniques attempt to print biological systems with ease and accuracy, however the clinical applications of in-vivo bioprinting are yet to be established. [68]

\section{D Printing}

3DBP has emerged as an effective platform for the delivery of gene and has also given rise to the possibility of $4 \mathrm{D}$ printing which measures the functionality of $3 \mathrm{D}$ printed structures in time. The geometry, functions, properties and mechanisms involved in 3DBP are also evaluated with different stimuli for development of controlled or sustained delivery systems. This application of stimuli-responsiveness and timedependence in 3DBP leads to 4D printing, wherein the structures show conformational changes when excited by different stimuli. 4D printing may carry a potential to further the applications of in-situ 3DBP and may help in establishing biological structures and scaffolds in-vivo. [71]

\section{Summary and Conclusions}

3D printing and bioprinting offer a method for artificial synthesis of biological structures or therapeutic delivery systems in cost- and time-effective manners. These methods encompass layer-by-layer deposition of biomaterials carefully chosen to closely resemble the desired assembly in tissues or cells. The ongoing research on bioprinting produces living tissues, bones, vascular structures and even whole organoids at the laboratory level for drug screening, analysis of various conditions and therapy. 3D bioprinting extends a great potential in gene modulation and gene delivery as it minimizes the tedious conventional production and cloning methods as well as enhances the targeted action while maintaining the integrity of the formed structures. Fabrication of nanostructures via 3D bioprinting for delivering therapeutic and genetic agents into the host cells offers applications in gene therapy with respect to tissue engineering, wound healing or skin regeneration, and especially in osteogenesis and genetic treatment of bone defects. Moreover, it aids in the development of personalized medicines for patient-specific conditions in carcinoma, inflammation, viral infections, etc. These techniques display potential benefits, however, extensive research is still required, since $3 \mathrm{D}$ printing has not yet been able to succeed in establishing high quality or fully functioning tissues and organs and the research conducted over the years are only at the preliminary or laboratory stage. This may be due to the complexities in the cellular structures of the human body and the immense network of specialized tissues, nerves and other components that are difficult to be replicated and positioned. Another problem is that the organelle structures and their constituents also differ from patient-to-patient, so maintaining or mimicking in-vivo environments becomes difficult, resulting in the slow progression of $3 \mathrm{D}$ bioprinting. The issues in selection of correct types of softwares, cells and bioinks suitable in function and printing widens the gap between experimental and clinical applications of 3D bioprinting. The compatibility of certain genes and other molecules along with their durability and viability with respect to the printing speed and applied pressure is an area of interest for the fabrication of functional structures and scaffolds. The sources used for the extraction of cells and genes for bioprinting applications also need to be regulated to maintain the level of purity and functionality. Furthermore, regulatory requirements pose as a challenge in the acceptance of $3 \mathrm{D}$ bioprinted structures. Nevertheless, with further advancements, establishment of clinical applications, development of in-situ and 4D printing models for bioprinting, real-time production of genes and other cell structures for diseased conditions may terminate the need for longterm therapies. Incorporation of modern technology such as artificial intelligence and Internet of Things (IoT), 3D bioprinting may emerge as one of the prominent techniques in the preparation of therapeutic delivery systems.

Author Contributions Dr. Pravin Shende: Conceptualization, writing (review and editing), supervision.

Riddhi Trivedi: Visualization, writing (original draft).

Data Availability Not applicable.

\section{Compliance with Ethical Standards}

Declaration of Conflict of Interest The authors declare that there are no conflicts of interest. 
Ethics Approval Not applicable.

Consent to Participate Not applicable.

Consent for Publication Not applicable.

Code Availability Not applicable.

\section{References}

1. Nelson, C, E., Duvall, C, L., Prokop, A., Gersbach, C, A., \& Davidson, J, M. (2020). Gene delivery into cells and tissues. In Principles of Tissue Engineering (pp. 519-554). Elsevier. https:// doi.org/10.1016/B978-0-12-818422-6.00030-7.

2. Cevher, E., Sezer, A, D., \& Çağlar, E, Ș. (2012). Gene delivery systems: Recent Progress in viral and non-viral therapy. In Recent Advances in Novel Drug Carrier Systems (pp. 437-470). https:// doi.org/10.5772/53392.

3. Grimm, D., \& Kay, M. A. (2007). RNAi and gene therapy: A mutual attraction. Hematology, 2007(1), 473-481. https://doi.org/ 10.1182/asheducation-2007.1.473.

4. Shende, P., \& Patel, C. (2019). siRNA: An alternative treatment for diabetes and associated conditions. Journal of Drug Targeting, 27(2), 174-182. https://doi.org/10.1080/1061186X.2018.1476518.

5. Peng, L.-H., Tsang, S.-Y., Tabata, Y., \& Gao, J.-Q. (2012). Genetically-manipulated adult stem cells as therapeutic agents and gene delivery vehicle for wound repair and regeneration. Journal of Controlled Release, 157(3), 321-330. https://doi.org/ 10.1016/j.jconrel.2011.08.027.

6. Shende, P., Gupta, H., \& Gaud, R. S. (2018). Cytotherapy using stromal cells: Current and advance multi-treatment approaches. Biomedicine \& Pharmacotherapy, 97, 38-44. https://doi.org/10. 1016/j.biopha.2017.10.127.

7. Phillips, A. J. (2001). The challenge of gene therapy and DNA delivery. Journal of Pharmacy and Pharmacology, 53(9), 11691174. https://doi.org/10.1211/0022357011776603.

8. Goswami, R., Subramanian, G., Silayeva, L., Newkirk, I., Doctor, D., Chawla, K., ... Betapudi, V. (2019). Gene therapy leaves a vicious cycle. Frontiers in Oncology, 9(APR), 1-25. https://doi. org/10.3389/fonc.2019.00297.

9. Yi, H.-G., Choi, Y.-J., Kang, K. S., Hong, J. M., Pati, R. G., Park, M. N., Shim, I. K., Lee, C. M., Kim, S. C., \& Cho, D.-W. (2016). A 3D-printed local drug delivery patch for pancreatic cancer growth suppression. Journal of Controlled Release, 238, 231-241. https:// doi.org/10.1016/j.jconrel.2016.06.015.

10. Beck, R. C. R., Chaves, P. S., Goyanes, A., Vukosavljevic, B., Buanz, A., Windbergs, M., Basit, A. W., \& Gaisford, S. (2017). 3D printed tablets loaded with polymeric nanocapsules: An innovative approach to produce customized drug delivery systems. International Journal of Pharmaceutics, 528(1-2), 268-279. https://doi.org/10.1016/j.ijpharm.2017.05.074.

11. Tagami, T., Yoshimura, N., Goto, E., Noda, T., \& Ozeki, T. (2019). Fabrication of Muco-adhesive Oral films by the 3D printing of Hydroxypropyl methylcellulose-based Catechin-loaded formulations. Biological and Pharmaceutical Bulletin, 42(11), 18981905. https://doi.org/10.1248/bpb.b19-00481.

12. Tagami, T., Ando, M., Nagata, N., Goto, E., Yoshimura, N., Takeuchi, T., Noda, T., \& Ozeki, T. (2019). Fabrication of Naftopidil-loaded tablets using a semisolid extrusion-type 3D printer and the characteristics of the printed hydrogel and resulting tablets. Journal of Pharmaceutical Sciences, 108(2), 907-913. https:// doi.org/10.1016/j.xphs.2018.08.026.
13. Wu, M., Zhang, Y., Huang, H., Li, J., Liu, H., Guo, Z., Xue, L., Liu, S., \& Lei, Y. (2020). Assisted 3D printing of microneedle patches for minimally invasive glucose control in diabetes. Materials Science and Engineering: C, 117, 111299. https://doi.org/10. 1016/j.msec.2020.111299.

14. Pravin, S., \& Sudhir, A. (2018). Integration of 3D printing with dosage forms: A new perspective for modern healthcare. Biomedicine \& Pharmacotherapy, 107(July), 146-154. https:// doi.org/10.1016/j.biopha.2018.07.167.

15. Mobaraki, M., Ghaffari, M., Yazdanpanah, A., Luo, Y., \& Mills, D. K. (2020). Bioinks and bioprinting: A focused review. Bioprinting, 18(August 2019), e00080. https://doi.org/10.1016/j.bprint.2020. $\mathrm{e} 00080$.

16. Liu, W., Li, Y., Liu, J., Niu, X., Wang, Y., \& Li, D. (2013). Application and performance of 3D printing in Nanobiomaterials. Journal of Nanomaterials, 2013, 1-7. https://doi.org/10.1155/ 2013/681050.

17. Gu, Z., Fu, J., Lin, H., \& He, Y. (2020). Development of 3D bioprinting: From printing methods to biomedical applications. Asian Journal of Pharmaceutical Sciences., 15, 529-557. https:// doi.org/10.1016/j.ajps.2019.11.003.

18. Lim, S. H., Kathuria, H., Tan, J. J. Y., \& Kang, L. (2018). 3D printed drug delivery and testing systems - A passing fad or the future? Advanced Drug Delivery Reviews, 132, 139-168. https:// doi.org/10.1016/j.addr.2018.05.006.

19. Zhang, B., Gao, L., Ma, L., Luo, Y., Yang, H., \& Cui, Z. (2019). 3D bioprinting: A novel avenue for manufacturing tissues and organs. Engineering, 5(4), 777-794. https://doi.org/10.1016/j.eng.2019.03. 009.

20. Bishop, E. S., Mostafa, S., Pakvasa, M., Luu, H. H., Lee, M. J., Wolf, J. M., Ameer, G. A., He, T. C., \& Reid, R. R. (2017). 3-D bioprinting technologies in tissue engineering and regenerative medicine: Current and future trends. Genes \& Diseases, 4(4), 185-195. https://doi.org/10.1016/j.gendis.2017.10.002.

21. Park, J. Y., Gao, G., Jang, J., \& Cho, D.-W. (2016). 3D printed structures for delivery of biomolecules and cells: Tissue repair and regeneration. Journal of Materials Chemistry B, 4(47), 7521-7539. https://doi.org/10.1039/C6TB01662F.

22. Vadodaria, S. S., He, Y., Mills, T., \& Wildman, R. (2020). Fabrication of surfactant-polyelectrolyte complex using valvejet 3D printing-aided colloidal self assembly. Colloids and Surfaces A: Physicochemical and Engineering Aspects, 600(March), 124914. https://doi.org/10.1016/j.colsurfa.2020.124914.

23. Yan, Y., Chen, H., Zhang, H., Guo, C., Yang, K., Chen, K., Cheng, R., Qian, N., Sandler, N., Zhang, Y. S., Shen, H., Qi, J., Cui, W., \& Deng, L. (2019). Vascularized 3D printed scaffolds for promoting bone regeneration. Biomaterials, 190-191, 97-110. https://doi.org/ 10.1016/j.biomaterials.2018.10.033.

24. Ashwin, B., Abinaya, B., Prasith, T. P., Chandran, S. V., Yadav, L. R., Vairamani, M., Patil, S., \& Selvamurugan, N. (2020). 3D-poly (lactic acid) scaffolds coated with gelatin and mucic acid for bone tissue engineering. International Journal of Biological Macromolecules, 162, 523-532. https://doi.org/10.1016/j. ijbiomac.2020.06.157.

25. Martin, V., Ribeiro, I. A., Alves, M. M., Gonçalves, L., Claudio, R. A., Grenho, L., Fernandes, M. H., Gomes, P., Santos, C. F., \& Bettencourt, A. F. (2019). Engineering a multifunctional 3Dprinted PLA-collagen-minocycline-nanoHydroxyapatite scaffold with combined antimicrobial and osteogenic effects for bone regeneration. Materials Science and Engineering: C, 101(March), 1526. https://doi.org/10.1016/j.msec.2019.03.056.

26. Zhang, B., Xue, Q., Li, J., Ma, L., Yao, Y., Ye, H., Cui, Z., \& Yang, H. (2019). 3D bioprinting for artificial cornea: Challenges and perspectives. Medical Engineering \& Physics, 71, 68-78. https://doi. org/10.1016/j.medengphy.2019.05.002. 
27. Ye, X., Li, L., Lin, Z., Yang, W., Duan, M., Chen, L., Xia, Y., Chen, Z., Lu, Y., \& Zhang, Y. (2018). Integrating 3D-printed $\mathrm{PHBV} /$ calcium sulfate hemihydrate scaffold and chitosan hydrogel for enhanced osteogenic property. Carbohydrate Polymers, 202, 106-114. https://doi.org/10.1016/j.carbpol.2018.08.117.

28. Lewicki, J., Bergman, J., Kerins, C., \& Hermanson, O. (2019). Optimization of 3D bioprinting of human neuroblastoma cells using sodium alginate hydrogel. Bioprinting, 16(May), e00053. https://doi.org/10.1016/j.bprint.2019.e00053.

29. Xu, X., Tao, J., Wang, S., Yang, L., Zhang, J., Zhang, J., Liu, H., Cheng, H., Xu, J., Gou, M., \& Wei, Y. (2019). 3D printing of nerve conduits with nanoparticle-encapsulated RGFP966. Applied Materials Today, 16, 247-256. https://doi.org/10.1016/j.apmt. 2019.05.014.

30. Vijayavenkataraman, S., Zhang, S., Thaharah, S., Sriram, G., Lu, W. F., \& Fuh, J. Y. H. (2018). Electrohydrodynamic jet 3D printed nerve guide conduits (NGCs) for peripheral nerve injury repair. Polymers, 10(7), 753. https://doi.org/10.3390/polym10070753.

31. Tao, J., Zhang, J., Du, T., Xu, X., Deng, X., Chen, S., et al. (2019). Rapid 3D printing of functional nanoparticle-enhanced conduits for effective nerve repair. Acta Biomaterialia, 90, 49-59. https://doi. org/10.1016/j.actbio.2019.03.047.

32. Mozetic, P., Giannitelli, S. M., Gori, M., Trombetta, M., \& Rainer, A. (2017). Engineering muscle cell alignment through 3D bioprinting. Journal of Biomedical Materials Research - Part A, 105(9), 2582-2588. https://doi.org/10.1002/jbm.a.36117.

33. Nishiguchi, A., Shima, F., Singh, S., Akashi, M., \& Moeller, M. (2020). 3D-printing of structure-controlled antigen nanoparticles for vaccine delivery. Biomacromolecules, 21(6), 2043-2048. https://doi.org/10.1021/acs.biomac.9b01775.

34. Chan, K., Wong, P.-Y., Parikh, C., \& Wong, S. (2018). Moving toward rapid and low-cost point-of-care molecular diagnostics with a repurposed 3D printer and RPA. Analytical Biochemistry, 545(12), 4-12. https://doi.org/10.1016/j.ab.2018.01.008.

35. Shende, P., Patil, A., \& Prabhakar, B. (2020). Layer-by-layer technique for enhancing physicochemical properties of actives. Journal of Drug Delivery Science and Technology, 56, 101519. https://doi. org/10.1016/j.jddst.2020.101519.

36. Paolini, A., Leoni, L., Giannicchi, I., Abbaszadeh, Z., D’Oria, V., Mura, F., Dalla Cort, A., \& Masotti, A. (2018). MicroRNAs delivery into human cells grown on 3D-printed PLA scaffolds coated with a novel fluorescent PAMAM dendrimer for biomedical applications. Scientific Reports, 8(1), 13888. https://doi.org/10.1038/ s41598-018-32258-9.

37. Cui, X., Dean, D., Ruggeri, Z. M., \& Boland, T. (2010). Cell damage evaluation of thermal inkjet printed chinese hamster ovary cells. Biotechnology and Bioengineering, 106(6), 963-969. https://doi. org/10.1002/bit.22762.

38. Xu, T., Rohozinski, J., Zhao, W., Moorefield, E. C., Atala, A., \& Yoo, J. J. (2009). Inkjet-mediated gene transfection into living cells combined with targeted delivery. Tissue Engineering - Part A, 15(1), 95-101. https://doi.org/10.1089/ten.tea.2008.0095.

39. Moncal, K. K., Aydin, R. S. T., Abu-Laban, M., Heo, D. N., Rizk, E., Tucker, S. M., Lewis, G. S., Hayes, D., \& Ozbolat, I. T. (2019). Collagen-infilled 3D printed scaffolds loaded with miR-148btransfected bone marrow stem cells improve calvarial bone regeneration in rats. Materials Science and Engineering C, 105(June), 110128. https://doi.org/10.1016/j.msec.2019.110128.

40. Abu Awwad, H. A. D. M., Thiagarajan, L., Kanczler, J. M., Amer, M. H., Bruce, G., Lanham, S., Rumney, R. M. H., Oreffo, R. O. C., \& Dixon, J. E. (2020). Genetically-programmed, mesenchymal stromal cell-laden \& mechanically strong 3D bioprinted scaffolds for bone repair. Journal of Controlled Release, 325(June), 335346. https://doi.org/10.1016/j.jconrel.2020.06.035.

41. Nagiah, N., Bhattacharjee, M., Murdock, C. J., Kan, H. M., Barajaa, M., \& Laurencin, C. T. (2020). Spatial alignment of 3D printed scaffolds modulates genotypic expression in pre-osteoblasts. Materials Letters, 276, 128189. https://doi.org/10.1016/j.matlet. 2020.128189.

42. Cunniffe, G. M., Gonzalez-Fernandez, T., Daly, A., Sathy, B. N., Jeon, O., Alsberg, E., \& Kelly, D. J. (2017). Three-dimensional bioprinting of Polycaprolactone reinforced gene activated bioinks for bone tissue engineering. Tissue Engineering Part A, 23(17-18), 891-900. https://doi.org/10.1089/ten.tea.2016.0498.

43. Gonzalez-Fernandez, T., Rathan, S., Hobbs, C., Pitacco, P., Freeman, F. E., Cunniffe, G. M., Dunne, N. J., McCarthy, H. O., Nicolosi, V., O'Brien, F. J., \& Kelly, D. J. (2019). Pore-forming bioinks to enable spatio-temporally defined gene delivery in bioprinted tissues. Journal of Controlled Release, 301(January), 13-27. https://doi.org/10.1016/j.jconrel.2019.03.006.

44. Sun, Y., You, Y., Jiang, W., Zhai, Z., \& Dai, K. (2019). 3Dbioprinting a genetically inspired cartilage scaffold with GDF5conjugated BMSC-laden hydrogel and polymer for cartilage repair. Theranostics, 9(23), 6949-6961. https://doi.org/10.7150/thno. 38061.

45. Loozen, L. D., Wegman, F., Öner, F. C., Dhert, W. J. A., \& Alblas, J. (2013). Porous bioprinted constructs in BMP-2 non-viral gene therapy for bone tissue engineering. Journal of Materials Chemistry B, 1(48), 6619-6626. https://doi.org/10.1039/c3tb21093f.

46. El-Ayoubi, R., Eliopoulos, N., Diraddo, R., Galipeau, J., \& Yousefi, A.-M. (2008). Design and fabrication of 3D porous scaffolds to facilitate cell-based gene therapy. Tissue Engineering Part A, 14(6), 1037-1048. https://doi.org/10.1089/ten.tea.2006.0418.

47. Chen, M., Andersen, M. Ø., Dillschneider, P., Chang, C.-C., Gao, S., Le, D. Q. S., et al. (2015). Co-delivery of siRNA and doxorubicin to cancer cells from additively manufactured implants. RSC Advances, 5(123), 101718-101725. https://doi.org/10.1039/ C5RA23748C.

48. Malecki, M., Kolsut, P., \& Proczka, R. (2005). Angiogenic and antiangiogenic gene therapy. Gene Therapy, 12, S159-S169. https://doi.org/10.1038/sj.gt.3302621.

49. Des Rieux, A., Ucakar, B., Mupendwa, B. P. K., Colau, D., Feron, O., Carmeliet, P., \& Préat, V. (2011). 3D systems delivering VEGF to promote angiogenesis for tissue engineering. Journal of Controlled Release, 150(3), 272-278. https://doi.org/10.1016/j. jconrel.2010.11.028.

50. Merceron, T. K., \& Murphy, S. V. (2015). Hydrogels for 3D bioprinting applications. In Essentials of $3 D$ biofabrication and translation. Inc.: Elsevier. https://doi.org/10.1016/B978-0-12800972-7.00014-1.

51. Tarassoli, S. P., Jessop, Z. M., Kyle, S., \& Whitaker, I. S. (2018). Candidate bioinks for 3D bioprinting soft tissue. In $3 D$ bioprinting for reconstructive surgery: Techniques and applications. Ltd.: Elsevier. https://doi.org/10.1016/B978-0-08-101103-4.00026-0.

52. Guo, T., Holzberg, T. R., Lim, C. G., Gao, F., Gargava, A., Trachtenberg, J. E., Mikos, A. G., \& Fisher, J. P. (2017). 3D printing PLGA: A quantitative examination of the effects of polymer composition and printing parameters on print resolution. Biofabrication, 9(2), 024101. https://doi.org/10.1088/1758-5090/ aa6370.

53. Thomas, A. M., Gomez, A. J., Palma, J. L., Yap, W. T., \& Shea, L. D. (2014). Heparin-chitosan nanoparticle functionalization of porous poly(ethylene glycol) hydrogels for localized lentivirus delivery of angiogenic factors. Biomaterials, 35(30), 8687-8693. https:// doi.org/10.1016/j.biomaterials.2014.06.027.

54. Müller, J., Jäkel, A. C., Schwarz, D., Aufinger, L., \& Simmel, F. C. (2020). Programming diffusion and localization of DNA signals in 3D-printed DNA-functionalized hydrogels. Small, 16(31), 2001815. https://doi.org/10.1002/smll.202001815.

55. Allen, P. B., Khaing, Z., Schmidt, C. E., \& Ellington, A. D. (2015). $3 \mathrm{D}$ printing with nucleic acid adhesives. ACS Biomaterials Science and Engineering, 1(1), 19-26. https://doi.org/10.1021/ab500026f. 
56. Li, Y., Bøtker, J., Rantanen, J., Yang, M., \& Bohr, A. (2020). In silico design and $3 \mathrm{D}$ printing of microfluidic chips for the preparation of size-controllable siRNA nanocomplexes. International Journal of Pharmaceutics, 583(December 2019), 119388. https:// doi.org/10.1016/j.ijpharm.2020.119388.

57. Salinas-Fernández, S., Santos, M., Alonso, M., Quintanilla, L., \& Rodríguez-Cabello, J. C. (2020). Genetically engineered elastinlike recombinamers with sequence-based molecular stabilization as advanced bioinks for 3D bioprinting. Applied Materials Today, 18, 100500. https://doi.org/10.1016/j.apmt.2019.100500.

58. Cambrian Genomics 3D Printing DNA - 3D Printing Industry. (n.d.). Retrieved January 5, 2021, from https://3dprintingindustry. com/news/cambrian-genomics-3d-printing-dna-25831/

59. 3ders.org - Synthesizing DNA 10,000 times cheaper with a DNA laser printer $3 \mathrm{D}$ Printer News \& 3D Printing News. (n.d.). Retrieved January 5, 2021, from http://www.3ders.org/articles/ 20130324-synthesizing-dna-times-cheaper-with-a-dna-laserprinter.html

60. DNA "Printing" A Big Boon To Research, But Some Raise Concerns : Shots - Health News : NPR. (n.d.). Retrieved January 5, 2021, from https://www.npr.org/sections/health-shots/2015/05/ 07/404460240/dna-printing-a-big-boon-to-research-but-someraise-concerns

61. Alvarez, C, H. (2015). DNA assembly and cloning in an overnight run with the BioXp ${ }^{\mathrm{TM}} 3200$ system. Nature Methods, 12(12), v-vi. https://doi.org/10.1038/nmeth.f.390.

62. Synthetic Genomics Inc. (n.d.). Incubated Technologies. Retrieved August 11, 2020, from https://syntheticgenomics.com/incubatedtechnologies/\#dna-printer

63. DNA Printer for Gene Production | NOVO Engineering. (n.d.). Retrieved January 5, 2021, from https://www.novoengineering. com/portfolio/dna-printer/

64. Martinez-Lage, M., Torres-Ruiz, R., \& Rodriguez-Perales, S. (2017). CRISPR/Cas9 technology: Applications and human disease modeling. In Progress in Molecular Biology and Translational Science (Vol. 152, pp. 23-48). Elsevier Inc. https:// doi.org/10.1016/bs.pmbts.2017.09.002.

65. Gopal, S., Rodrigues, A. L., \& Dordick, J. S. (2020). Exploiting CRISPR Cas9 in three-dimensional stem cell cultures to model disease. Frontiers in Bioengineering and Biotechnology, 8(June), 1-15. https://doi.org/10.3389/fbioe.2020.00692.

66. Reuters. (n.d.). Tesla to make molecule printers for German COVID-19 vaccine developer CureVac - Reuters. Retrieved August 11, 2020, from https://www.reuters.com/article/us-healthcoronavirus-tesla/musk-says-tesla-is-building-rna-microfactoriesfor-curevac-idUSKBN243168

67. CureVac Receives Regulatory Approval from German and Belgian Authorities to Initiate Phase 1 Clinical Trial of its SARS-CoV-2 Vaccine Candidate | English. (n.d.). Retrieved August 11, 2020, from https://www.curevac.com/news/curevac-receives-regulatoryapproval-from-german-and-belgian-authorities-to-initiate-phase-1clinical-trial-of-its-sars-cov-2-vaccine-candidate

68. Singh, S., Choudhury, D., Yu, F., Mironov, V., \& Naing, M, W. (2020). In situ bioprinting - Bioprinting from benchside to bedside? Acta Biomaterialia, 101(xxxx), 14-25. https://doi.org/10.1016/j. actbio.2019.08.045

69. Kérourédan, O., Hakobyan, D., Rémy, M., Ziane, S., Dusserre, N., Fricain, J. C., Delmond, S., Thébaud, N. B., \& Devillard, R. (2019). In situ prevascularization designed by laser-assisted bioprinting: Effect on bone regeneration. Biofabrication, 11(4), 045002. https://doi.org/10.1088/1758-5090/ab2620.

70. Albanna, M., Binder, K. W., Murphy, S. V., Kim, J., Qasem, S. A., Zhao, W., Tan, J., el-Amin, I. B., Dice, D. D., Marco, J., Green, J., Xu, T., Skardal, A., Holmes, J. H., Jackson, J. D., Atala, A., \& Yoo, J. J. (2019). In situ bioprinting of autologous skin cells accelerates wound healing of extensive excisional full-thickness wounds. Scientific Reports, 9(1), 1856. https://doi.org/10.1038/s41598018-38366-w.

71. Ashammakhi, N., Ahadian, S., Zengjie, F., Suthiwanich, K., Lorestani, F., Orive, G., Ostrovidov, S., \& Khademhosseini, A. (2018). Advances and future perspectives in 4D bioprinting. Biotechnology Journal, 13(12), 1-21. https://doi.org/10.1002/biot. 201800148 .

Publisher's Note Springer Nature remains neutral with regard to jurisdictional claims in published maps and institutional affiliations. 